\section{Neutropenie bei Brustkrebs: Prophylaxe auch bei geringem Risiko?}

\author{
Manche Chemotherapieregimes gehen nur mit einem geringen Risiko für \\ eine febrile Neutropenie (FN) einher. Ob dann trotzdem eine Prophylaxe \\ mit G-CSF sinnvoll ist, haben dänische Wissenschaftler bei Brustkrebs- \\ patientinnen retrospektiv untersucht.
}

$\mathrm{N}$ eutropenie ist eine schwerwiegende Nebenwirkung von Chemotherapien. Manche Regime (z.B. Docetaxel/ Cyclophosphamid) gelten als nur milde, andere wie Doxorubicin/Cyclophosphamid sogar als wenig Neutropenie-auslösend. Richtlinien empfehlen den Einsatz von Granulozyten-Kolonie-stimulierenden Wachstumsfaktoren (G-CSF) als Prophylaxe erst ab einem FN-Risiko von mindestens $20 \%$. Ob auch die Patienten mit einem niedrigeren Neutropenierisiko profitieren, haben dänische Forscher jetzt für 3 häufig benutzte Chemotherapieregime mit niedrigem bis mittlerem Neutropenierisiko untersucht. Die Daten von 8.745 Brustkrebs- patientinnen wurden aus US-amerikanischen Versicherungsdatenbanken entnommen. Sie hatten zwischen 2008 und 2013 eine Chemotherapie bekommen: Docetaxel/Cyclophosphamid (TC; $\mathrm{n}=$ 4.815), Docetaxel/Carboplatin/Trastuzumab (TCH; $\mathrm{n}=2.292$ ) oder Doxorubicin/Cyclophosphamid (AC; $\mathrm{n}=1.638$ ). Eine primäre Prophylaxe galt als G-CSFGabe innerhalb von 5 Tagen nach Beginn der Chemotherapie. Endpunkte waren Neutropenie, Fieber oder eine infektionsbedingte Hospitalisierung binnen 21 Tagen.

Mehr als die Hälfte der Patientinnen erhielt eine Prophylaxe. Eine stationär behandlungsbedürftige FN entwickelte sich mit versus ohne Prophylaxe mit der folgenden Häufigkeit: 2,0 versus 7,1\% bei TC (Odds Ratio [OR] 0,29; $\mathrm{p}=0,001$ ), 1,3 versus $7,1 \%$ bei TCH (OR 0,19; $\mathrm{p}=0,001)$ und 4,7 versus $3,8 \%$ bei $\mathrm{AC}$ (OR 1,$21 ; \mathrm{p}=0,41$ )

20 Patientinnen aus der TC-Gruppe müssten also für 21 Tage eine G-CSFProphylaxe bekommen, um eine Neutropenie-bedingte Hospitalisierung $\mathrm{zu}$ verhindern; mit dem TCH-Regime wären es 18 Patientinnen.

Fazit: Eine primäre G-CSF-Prophylaxe ist mit einem niedrigen bis moderaten Nutzen bei Patientinnen mit Brustkrebs assoziiert, die eine TC- oder eine TCHChemotherapie erhalten. Eine weitere Evaluation ist nötig, um besser zu verstehen, welche Patientinnen am meisten von einer G-CSF-Prophylaxe profitieren.

Christina Berndt

Agiro A et al. Risk of Neutropenia-Related Hospitalization in Patients Who Received Colony-Stimulating FactorsWith Chemotherapy for Breast Cancer. J Clin Oncol. 2016 Sep 19. [Epub ahead of print]

\title{
Früher Brustkrebs: Alle postoperativ bestrahlen?
}

\section{Nach brusterhaltender Operation des Mammakarzinoms wird meist bestrahlt, um das Risiko für ein ipsi- laterales Rezidiv zu senken. Doch ist das bei allen Patientinnen nötig?}

\footnotetext{
D ie Mehrheit der Patientinnen mit T1-2 N0-Tumoren wird heute brusterhaltend operiert und anschließend bestrahlt. 2 Metaanalysen der Early Breast Cancer Trialists' Collaborative Group (EBCTCG) bestätigten, dass die postoperative Radiotherapie (RT) das Risiko für ein ipsilaterales Rezidiv senkt. Dennoch bleibt die Frage offen, ob wirklich alle Patientinnen davon profitieren.

In der 15-Jahres-Analyse einer schwedischen Studie zum landesweiten Mammografie-Screeningprogramm hatten 1.187 Patientinnen (median 60 Jahre, $78 \%$ postmenopausal) mit $\mathrm{T} 1-1 \mathrm{~N} 0$ Mammakarzinom zwischen 1991 und 1997 nach einer brusterhaltenden Thera-
}

pie randomisiert eine postoperative RT oder keine lokale Therapie erhalten. Bei Stadium-II-Tumoren war zudem eine adjuvante systemische Therapie angeboten worden. $65 \%$ der Tumoren waren beim Screening entdeckt worden. Die mediane Tumorgröße betrug $12 \mathrm{~mm}$, im Median waren 11 Lymphknoten untersucht worden, $57 \%$ der Tumoren waren Östrogenrezeptor(ER)-positiv, $13 \%$ ERnegativ.

Nach 15 Jahren wurden in der Kontrollgruppe signifikant mehr ipsilaterale Rezidive (23,9 vs. $11,5 \%$; $\mathrm{p}<0,001)$ und ein schlechteres rezidivfreies Überleben festgestellt (51,7 vs. $60,4 \%$; $=0,0013)$. Der Haupteffekt der RT war während der ersten 5 Jahre zu beobachten.

Bei vorab als Subgruppe mit niedrigem Risiko definierten Patientinnen jenseits des 64. Lebensjahres mit ER- und Progesteronrezeptor-positiven Tumoren $<21 \mathrm{~mm}$ zeigte sich ohne RT eine kumulative Inzidenz ipsilateraler Rezidive nach 15 Jahren von $25,9 \%$, nach RT von $5,3 \%$. Der Effekt der RT war bei ihnen somit stärker als in der Gesamtkohorte. In den ersten 5 Jahren trat bei diesen $\mathrm{Pa}$ tientinnen nach RT kein einziges ipsilaterales Rezidiv auf.

Auf die Mortalität wirkte sich die RT jedoch nicht aus: Die Rate für das Gesamtüberleben nach 15 Jahren war in der Kontrollgruppe nicht signifikant niedriger als in der RT-Gruppe $(68,4$ vs. $71,1 \%$; $\mathrm{p}=0,68$ ), auch war die brustkrebsspezifische Mortalität nicht höher.

Fazit: Die RT nach brusterhaltender Operation reduziert in den ersten 15 Jahren die Inzidenz ipsilateraler Rezidive beim frühen Mammakarzinom. Subgruppen, bei denen man auf die RT verzichten könnte, ließen sich in der Studie nicht identifizieren. Kathrin von Kieseritzky

Killander F et al. No breast cancer subgroup can be spared postoperative radiotherapy after breast-conserving surgery. Fifteen-year results from the Swedish Breast Cancer Group randomised trial, SweBCG 91 RT. Eur J Cancer. 2016;67:57-65. 